\title{
IncRNA and mRNA sequencing of the left testis in experimental varicocele rats treated with Morinda officinalis polysaccharide
}

\author{
LIHONG ZHANG $^{1-3}$, XIAOZHEN ZHAO ${ }^{1-3}$ and WEI WANG ${ }^{1-3}$ \\ ${ }^{1}$ Department of Human Anatomy, Histology and Embryology, School of Basic Medical Sciences, Fujian Medical University; \\ ${ }^{2}$ Key Laboratory of Aging and Neurodegenerative Disease, School of Basic Medical Sciences, Fujian Medical University; \\ ${ }^{3}$ Laboratory of Clinical Applied Anatomy, Fujian Medical University, Fuzhou, Fujian 350108, P.R. China
}

Received May 20, 2020; Accepted July 6, 2021

DOI: $10.3892 /$ etm.2021.10570

\begin{abstract}
Varicocele is a common disease of the male reproductive system. Morinda (M.) officinalis is a Chinese herbal medicine, whose main bioactive component $M$. officinalis polysaccharide (MOP) is believed to have a therapeutic effect on varicocele; however, the underlying molecular mechanisms of this effect are poorly understood. In the present study, 24 rats were randomly divided into three groups: i) Control group; ii) experimental varicocele group; and iii) $300 \mathrm{mg} / \mathrm{kg} \mathrm{MOP}$ administration group. Analysis of mRNA and long non-coding RNA (lncRNA) expression in rat left testicular tissue was performed. The results suggested that a total of $144 \mathrm{mRNAs}$ and 63 lncRNAs, 63 mRNAs and 148 lncRNAs, and 173 mRNAs and 54 lncRNAs were differentially expressed between the varicocele non-treatment and control groups, the varicocele treatment and varicocele non-treatment groups, and the varicocele treatment and control groups, respectively. Following validation by reverse transcription-quantitative PCR, the Yip1 domain family member 7 (YIPF7) gene was identified as a key mediator of varicocele pathogenesis and repair effect of MOP. Additionally, genes such as purinergic receptor P2X 4 (P2RX4), transmembrane protein 225B (TMEM255B) and Wnt family member 9B (WNT9B) were confirmed to be differentially expressed between the varicocele non-treatment
\end{abstract}

Correspondence to: Professor Wei Wang or Professor Xiaozhen Zhao, Department of Human Anatomy, Histology and Embryology, School of Basic Medical Sciences, Fujian Medical University, 1 Xuefu North Road, University Town, Fuzhou, Fujian 350108, P.R. China

E-mail: 1401411234@qq.com

E-mail: zxz8338@163.com

Abbreviations: MOP, Morinda officinalis polysaccharide; VC, varicocele; lncRNA, long non-coding RNA; TJ, tight junction; RNA-seq, RNA sequencing; BTB, blood-testis barrier; DE, differentially expressed; RT-qPCR, reverse transcription-quantitative PCR reaction; GO, Gene Ontology; KEGG, Kyoto Encyclopedia of Genes and Genomes

Key words: experimental varicocele, Morinda officinalis polysaccharide, mRNA sequencing, lncRNA sequencing and control groups. We hypothesize that TMEM255B could be a potential novel diagnostic biomarker for varicocele; WNT9B and $P 2 R X 4$ likely play notable roles in the pathophysiology of the disease through the Wnt signaling pathway and regulation of transmembrane ion channels, respectively. In summary, the present study delineated the molecular mechanisms underlying varicocele pathogenesis and the therapeutic effect of MOP, identified a potential novel diagnostic marker and therapeutic target for varicocele, and provided feasible directions for further studies in the future.

\section{Introduction}

Varicocele, an abnormal varix of the pampiniform plexus vein, is the most common cause of male infertility. The global incidence of varicocele is $15-20 \%$ in the general population and $\sim 40 \%$ in patients who are infertile (1-4); this may have a negative impact on human evolution. For spermatogenesis to occur, the testis must descend into the scrotum during the embryonic period to provide proper the conditions of a temperature $\sim 2^{\circ} \mathrm{C}$ lower than the central body temperature (5). The upright human posture requires spermatic veins to work against gravity to return deoxygenated blood back to the heart (6). If the valves inside these veins fail, gravity can make the blood pool inside the testicle, eventually leading to enlargement of the veins and formation of a varicocele (7). In $\sim 90 \%$ of cases, the disease occurs on the left side, due to the longer left testicular vein, hemodynamics and higher incidence of abnormal venous valves $(8,9)$. Effective therapies for varicocele have yet to be determined $(8,10-12)$. The experimental rat varicocele model, first established in 1981 (13) by partly ligating the left renal vein, is widely used to investigate the pathophysiology, diagnosis and treatment of the disease. Researchers have shown that the following mechanisms contribute to the pathogenesis of varicocele: i) Neuroendocrine system dysfunction; ii) hypoxia; iii) accumulation of metabolites and toxicants; iv) oxidative stress; v) disruption of the blood-testis barrier (BTB); and vi) cell damage resulting from increased testicular temperature $(14,15)$.

In China, Morinda officinalis F.C.How grows in the Guangdong, Guangxi and Fujiang provinces. The roots of M. officinalis are used in a Chinese herbal medicine known as Bajitian; it has a long history of use for the improvement of 
male sexual function and the treatment of male reproductive system defects in China (16). M. officinalis polysaccharide (MOP) is one of the main active components of M. officinalis. Our previous research found that MOP can repair varicoceleinduced damage to the male rat reproductive system by promoting spermatogenesis, reconstructing the BTB, increasing the expression of tight junction (TJ) proteins and restoring hormonal balance, with $300 \mathrm{mg} / \mathrm{kg}$ being the most effective dosage (17). However, the molecular mechanisms underlying the pathophysiology of varicocele and the physiological functions and therapeutic effects of MOP in varicocele are yet to be explored in detail.

Advances in high-throughput RNA sequencing (RNA-Seq) technology and bioinformatics analysis methods have enabled researchers to gain insight into the nature of diseases at the RNA level by studying the dynamics of mRNA, microRNA and long non-coding RNA (lncRNA) (18) expression levels. According to previous research, lncRNAs, which are $>200$ nucleotides long and found in multiple organisms (19), play notable roles at almost every step of gene expression and take part in various disease processes (20); for example, in Parkinson's disease (21), leukemia (22), diabetes (23), cardiovascular disease $(24)$, colon cancer $(25,26)$, lung cancer $(27)$ and prostate cancer (28). Due to their diverse bioactivities, lncRNAs are regarded as potential targets for the diagnosis and treatment of varicocele.

In the present study, rats with surgically induced varicocele were treated with either saline or $300 \mathrm{mg} / \mathrm{kg}$ MOP by gavage. Identification of differentially expressed (DE) mRNAs and IncRNAs in rat left testicular tissue was conducted by RNA-Seq, and the results were verified by reverse transcription-quantitative PCR (RT-qPCR). Bioinformatics resources, such as Gene Ontology (GO), Kyoto Encyclopedia of Genes and Genomes (KEGG) pathway analysis and co-expression network analysis, were utilized to explore the mechanisms underlying varicocele pathophysiology and the therapeutic effect of MOP, in addition to the interactions between DE mRNAs and lncRNAs in varicocele. The aim of the current study was to identify novel targets and methods for varicocele diagnosis and therapy, and establish a theoretical basis for the use of MOP in clinical practice.

\section{Materials and methods}

Extraction of MOP. The plant M. officinalis used in the present study was grown (via artificial cultivation) in the Nanjing county of Fujian province in China. MOP was extracted from dried root according to a previously described protocol $(17,29,30)$. In brief, after being ground, the dried root was boiled in ethyl alcohol to remove oligosaccharides and lipids, extracted using deionized water and precipitated with $60 \%$ alcohol; thereafter, Sevag reagent (Sinopharm Chemical Reagent Co., Ltd) was added to remove the protein.

Experimental design. Male, 6- to 7-week-old Sprague-Dawley rats, weighing $200 \pm 10 \mathrm{~g}$, were purchased from and kept in The Laboratory Animal Center of Fujian Medical University (Fuzhou, China). All rats were housed in normal atmosphere $\left(\mathrm{N}_{2}, 78 \% ; \mathrm{O}_{2}, 21 \% ; \mathrm{CO}_{2}, 0.03 \%\right)$ and specific pathogen-free controlled environmental conditions, with a temperature of $\sim 23^{\circ} \mathrm{C}$, a 12 -h day/night cycle, a humidity of $40-70 \%$ and free access to standard rat food and water. All procedures conformed to the Guidelines for the Care and Use of Laboratory Animals established by Fujian Medical University. The study was approved by The Animal Approval Committee of Fujian Medical University (approval no. SYXK-2012-0001).

A total of 24 rats were randomly divided into three equal groups: Control group, varicocele non-treatment group (VC) and varicocele treatment group $(\mathrm{VC}+\mathrm{MOP})$. Induction of varicocele was attempted by partial ligation of the left renal vein under anesthesia using $30 \mathrm{mg} / \mathrm{kg}$ sodium pentobarbital by intraperitoneal injection (31). Control rats underwent the same operation without the partial ligation, and were fed a conventional diet for 12 weeks. Subsequently, 8 weeks post-surgery, rats in the $\mathrm{VC}$ and $\mathrm{VC}+\mathrm{MOP}$ groups were given a daily oral gavage of $2 \mathrm{ml}$ normal saline or $300 \mathrm{mg} / \mathrm{kg}$ MOP, respectively, for 4 weeks; rats in the Control group were given nothing except conventional diet. The rats (weight, $500 \pm 25 \mathrm{~g}$ ) were sacrificed by cervical dislocation after being anesthetized using $30 \mathrm{mg} / \mathrm{kg}$ sodium pentobarbital by intraperitoneal injection to collect left testicular tissue, which was immediately placed in liquid nitrogen. Varicocele rats in which vascular dilation did not occur or left kidney atrophy occurred were excluded from the follow-up experiment.

mRNA and lncRNA sequencing analysis. RNA-Seq analysis was performed by Kangchen BioTech Co., Ltd. The process used was divided into three parts: i) Extraction of total RNA from rat left testicular tissue; ii) construction of a cDNA library; and iii) RNA sequencing.

In brief, total RNAs were extracted using TRIzol ${ }^{\circledR}$ Reagent (Takara Bio Europe SAS) according to the manufacturer's instructions, followed by purification by Ribo-Zero ${ }^{\mathrm{TM}}$ Magnetic Gold Kit (Human/Mouse/Rat) (cat. no. MRZG12324; Epicentre, Illumina, Inc.). The quantity, integrity and concentration of the extracted RNAs were verified by $1 \%$ agarose gel electrophoresis and NanoDrop ND-1000 spectrophotometry (Thermo Fisher Scientific, Inc.). For library preparation, the KAPA Stranded RNA-Seq Library Prep kit (cat. no. KK8401; Illumina, Inc.) was used (paired-ended sequencing; 500 bp), with 1-2 $\mu \mathrm{g}$ of sample RNA. The established libraries were examined using an Agilent 2100 Bioanalyzer G2938C (Agilent Technologies, Inc.) and quantified by RT-qPCR. The samples were denatured to single-stranded DNA with $0.1 \mathrm{M} \mathrm{NaOH}$ (final density, $8 \mathrm{pM}$; concentrations measured by RT-qPCR) and amplified using TruSeqSR Cluster Kit v3-cBot-HS (cat. no. GD-401-3001; Illumina, Inc.). The libraries were pooled and sequenced on the Illumina HiSeq 4000 system (Illumina, Inc.) using 150 cycles of paired-end sequencing.

The quality of the raw sequencing data was assessed using FastQC software v0.11.7 (http://www.bioinformatics. babraham.ac.uk/projects/fastqc/), before the adapter sequences and poor quality bases were trimmed from the reads using the Cutadapt software v1.17 (http://dx.doi. org/10.14806/ej.17.1.200) (32). The Hisat2 software v2.0.4 (http://ccb.jhu.edu/software/hisat2) and StringTie software v1.2.2 (http://ccb.jhu.edu/software/stringtie) (33) were used to calculate fragments per kilobase of transcript per million mapped reads (FPKM) values, which represented the final expression levels of the genes. Differential gene expression 
level analyses were performed using the Ballgown package in R v2.10.0 (https://www.r-project.org) (32). An average FPKM value $>0.5$ was chosen as the cut-off for gene expression in the samples.

$R T$ - $q P C R$. To confirm the results of RNA-Seq, specific DE patterns were validated by RT-qPCR, with three biological replicates for each group. TRIzol ${ }^{\circledR}$ reagent (Invitrogen; Thermo Fisher Scientific, Inc.) was used to extract the total RNAs from rat testicular tissue, according to the manufacturer's instructions. cDNA was synthesized using $3 \mu \mathrm{g}$ total RNAs, SuperScript III reverse transcriptase (Invitrogen; Thermo Fisher Scientific, Inc.), 5X reverse transcription buffer solution (Invitrogen; Thermo Fisher Scientific, Inc.), $2.5 \mathrm{mM}$ dNTPs (HyTest, Ltd), primers (Invitrogen; Thermo Fisher Scientific, Inc.), and the GeneAmp PCR 9700 System $\left(50^{\circ} \mathrm{C}\right.$ for $60 \mathrm{~min}$, $70^{\circ} \mathrm{C}$ for $15 \mathrm{~min}$ and stored at $4^{\circ} \mathrm{C}$ for infinite time; Applied Biosystems; Thermo Fisher Scientific, Inc.). The RT-qPCR analysis was performed using the $\mathrm{SYBR}^{\circledR}$-Green Real-time PCR Master mix (Arraystar, Inc.) and ViiA ${ }^{\mathrm{TM}} 7$ Real-time PCR system (Applied Biosystems; Thermo Fisher Scientific, Inc.), with three technical replicates set for each sample. The thermocycling conditions were as follows: $95^{\circ} \mathrm{C}$ for $10 \mathrm{~min}$; $95^{\circ} \mathrm{C}$ for $10 \mathrm{sec}$, then $60^{\circ} \mathrm{C}$ for $1 \mathrm{~min}(40$ cycles $) ; 95^{\circ} \mathrm{C}$ for $10 \mathrm{sec} ; 60^{\circ} \mathrm{C}$ for $1 \mathrm{~min}$; and $95^{\circ} \mathrm{C}$ for $15 \mathrm{sec}$. The primers were designed and purchased from Kangchen BioTech Co., Ltd. The primer sequences are shown in Table I. The relative expression of each gene was quantified by comparing its $\mathrm{Cq}$ value $\left(2^{-\Delta \Delta \mathrm{Cq}}\right.$ method) with that of the housekeeping gene, $\beta$-actin (34).

Statistical analyses. RNA-Seq and RT-qPCR were performed in three independent biological replicates for each group. Only genes with an absolute fold-change $>1.2$ and P-value $\leq 0.05$ were considered to be differentially expressed between the groups. The data were analyzed in SPSS v21.0 (IBM Corp.), and the results are presented as the mean \pm standard deviation. Pearson's correlation was used in scatter plot of DE genes. One-way ANOVA was used to compare gene expression levels between two groups, and $\mathrm{P} \leq 0.05$ was considered to indicate a statistically significant difference.

Bioinformatic analyses. GO classification and KEGG pathway enrichment analyses were performed using the website of Gene Ontology Resource (http://www.geneontology.org) and KEGG pathway database (https://www.kegg.jp/kegg/pathway. $\mathrm{html}$ ) respectively. The co-expression of DE mRNA (coding genes) and lncRNA (non-coding genes) networks were visualized using Cytoscape v2.8.3 (http://cytoscape.org). The present study data were uploaded to the Gene Expression Omnibus (GEO) website (https://www.ncbi.nlm.nih.gov/geo/; accession no. GSE139447; secure token: sfaxocskhpoxnix).

\section{Results}

Identification of DE mRNAs and IncRNAs. The present study data were uploaded to the Gene Expression Omnibus website. The top 10 upregulated and downregulated DE genes (including mRNAs and lncRNAs) are listed in Tables II and III, respectively. Hierarchical cluster (Fig. 1), scatter plot (Fig. 2) and volcano plot (Fig. 3) analyses of the
Table I. Quantitative PCR primer sequences.

Gene $\quad$ Primer sequence, 5'-3'

\section{YIPF7}

Forward

Reverse

WNT9B

Forward

Reverse

TMEM255B

Forward

Reverse

P2RX4

Forward

Reverse

GPR162

Forward

Reverse

CLSTN2

Forward

Reverse

AKRlB8

Forward

Reverse

NILR1

Forward

Reverse

AABR07007833.1

Forward

Reverse

AABR07014649.1

Forward

Reverse

AABR07050146.1

Forward

Reverse

AABR07058711.1

Forward

Reverse

AABR07069067.1

Forward

Reverse

AC125688.1

Forward

Reverse

AABR07004428.1

Forward

Reverse

$\beta$-ACTIN

Forward

Reverse
ATAATGATTCTAATGCTTACGGA ACAAGAACATCTCTGGTGGAAC

GTGTGTGGTGACAACCTGAAGTA TGACACGCCATGACACTTGC

GCTTGTGCCCTCCGTCTATGA AGGGCTGTAGTGGCAGAGGGT

ATATTCCGTCTTGGCACAATC CTCTATCCAGGTTGCAGTCCC

F:TTGCCGTGGAAACCTTGGTG R:CCTAAGCCCATTTCTCCTGCC

TCAAAGAACCAGCCTACAAAG AAGCAGTTACCAGGATCTCATAC

CAGTTGAGCGACCAGGAGATG CTGCGTCATAGGGAAACTCTT

CCAGGAGGAAAGCGTTTATGC GGGTTTTACTTGGGCGTATGTCT

GTGTTCACTACCTCATTCCGTC TCCTGCTCCTCTTGGTTCTTA

TGATGAGAAGACTATGAAGAATGC AAGTTTGGTTTGAATGCTGC

CTGAGGCAAAGGGACTCTGTA CTTTGTTGGAGGGACAGCTC

TGCTCCATAAGTATCGAAGGC CAGATATTAGCCACAAACCTCA

GCAGCTTCTTGACATCACATT A TAGGCATTCCTTAGAGCATTT

CTATTCATAGCACCTCTGTGCTG CGTGGAGTTCTCTGATCTTTGTA

CACCCACGGAGATCCCACT
GAGCCTTCCCTGTAGCTGGTT

CGAGTACAACCTTCTTGCAGC ACCCATACCCACCATCACAC
AKR1B8, Aldo-keto reductase family 1, member B8; CLSTN2, calsyntenin 2; GPR162, G protein-coupled receptor 162; NILR1, leukocyte immunoglobulin like receptor B2; P2RX4, purinergic receptor P2X 4; TMEM255B, transmembrane protein 225B; WNT9B, Wnt family member 9B; YIPF7, Yip1 domain family member 7. 
Table II. Top 10 upregulated differentially expressed genes between groups by RNA-sequencing analysis.

A, VC vs. Control

\begin{tabular}{|c|c|c|c|c|}
\hline Tract ID & Gene name & Gene type & Fold-change & P-value \\
\hline ENSRNOG00000026336 & TMEM255B & Protein-coding & 7.72 & $6.9 \times 10^{-4}$ \\
\hline ENSRNOG00000006494 & $T U B G 2$ & Protein coding & 2.68 & $7.4 \times 10^{-3}$ \\
\hline ENSRNOG00000054954 & NILR1 & Protein coding & 2.33 & $3.2 \times 10^{-2}$ \\
\hline ENSRNOG00000026497 & $P I G C$ & Protein coding & 2.26 & $7.5 \times 10^{-4}$ \\
\hline ENSRNOG00000051890 & LOC 102549170 & lincRNA & 2.09 & $1.7 \times 10^{-2}$ \\
\hline ENSRNOG00000016143 & GPR162 & Protein coding & 1.91 & $1.1 \times 10^{-3}$ \\
\hline ENSRNOG00000053415 & AABR07014649.1 & lincRNA & 1.74 & $1.2 \times 10^{-2}$ \\
\hline ENSRNOG00000058993 & AABR07062183.1 & Protein coding & 1.70 & $9.7 \times 10^{-3}$ \\
\hline ENSRNOG00000002207 & GUF1 & Protein coding & 1.69 & $3.7 \times 10^{-2}$ \\
\hline ENSRNOG00000052173 & $R A N B P 3 L$ & Protein coding & 1.61 & $1.4 \times 10^{-3}$ \\
\hline
\end{tabular}

$\mathrm{B}, \mathrm{VC}+\mathrm{MOP}$ vs. VC

\begin{tabular}{|c|c|c|c|c|}
\hline Tract ID & Gene name & Gene type & Fold-change & P-value \\
\hline ENSRNOG00000029401 & $A C T G 2$ & Protein coding & 3.04 & $3.0 \times 10^{-2}$ \\
\hline ENSRNOG00000017669 & CHDIL & Protein coding & 2.30 & $1.3 \times 10^{-2}$ \\
\hline ENSRNOG00000061865 & AABR07019334.1 & lincRNA & 2.05 & $5.3 \times 10^{-3}$ \\
\hline ENSRNOG00000060166 & AABR07069067.1 & lincRNA & 1.76 & $3.1 \times 10^{-2}$ \\
\hline ENSRNOG00000059729 & $A C 116236.2$ & lincRNA & 1.63 & $1.2 \times 10^{-2}$ \\
\hline ENSRNOG00000007335 & CCL11 & Protein coding & 1.58 & $3.2 \times 10^{-2}$ \\
\hline ENSRNOG00000057837 & AABR07017236.1 & Protein coding & 1.57 & $8.9 \times 10^{-3}$ \\
\hline ENSRNOG00000057135 & $A A B R 07070714.2$ & lincRNA & 1.56 & $4.4 \times 10^{-2}$ \\
\hline ENSRNOG00000042665 & AABR07007130.2 & Protein coding & 1.56 & $2.8 \times 10^{-2}$ \\
\hline ENSRNOG00000052005 & AABR07028945.1 & lincRNA & 1.55 & $1.6 \times 10^{-2}$ \\
\hline
\end{tabular}

$\mathrm{C}, \mathrm{VC}+\mathrm{MOP}$ vs. Control

\begin{tabular}{|c|c|c|c|c|}
\hline Tract ID & Gene name & Gene type & Fold-change & P-value \\
\hline ENSRNOG00000043085 & CLSTN2 & Protein coding & 2.11 & $2.4 \times 10^{-2}$ \\
\hline ENSRNOG00000023861 & SNAP91 & Protein coding & 2.08 & $2.9 \times 10^{-2}$ \\
\hline ENSRNOG00000059602 & AABR07072984.1 & lincRNA & 1.73 & $4.5 \times 10^{-2}$ \\
\hline ENSRNOG00000059504 & AABR07015078.2 & Protein coding & 1.67 & $2.9 \times 10^{-2}$ \\
\hline ENSRNOG00000052211 & AABR07058167.1 & lincRNA & 1.65 & $6.2 \times 10^{-3}$ \\
\hline ENSRNOG00000052668 & TCF24 & Protein coding & 1.63 & $3.9 \times 10^{-2}$ \\
\hline ENSRNOG00000057070 & $A A B R 07014350.1$ & lincRNA & 1.63 & $5.4 \times 10^{-3}$ \\
\hline ENSRNOG00000034129 & $A A B R 07061382.1$ & Protein coding & 1.51 & $4.5 \times 10^{-2}$ \\
\hline ENSRNOG00000023035 & SMIM8 & Protein coding & 1.50 & $6.3 \times 10^{-3}$ \\
\hline ENSRNOG00000011969 & DOCK9 & Protein coding & 1.48 & $1.2 \times 10^{-4}$ \\
\hline
\end{tabular}

lincRNA, long intragenic non-coding RNA; VC, varicocele; MOP, Morinda officinalis polysaccharide; TMEM255B, transmembrane protein 225B; TUBG2, tubulin $\gamma$-2 chain; NILR1, leukocyte immunoglobulin-like receptor B2; PIGC, phosphatidylinositol glycan anchor biosynthesis class C; GPR162, G protein-coupled receptor 162; GUF1, GUF1 homolog, GTPase; RANBP3L, RAN binding protein 3-like; ACTG2, Actin, g smooth muscle; CHD1L, chromodomain-helicase-DNA-binding protein 1-like; CCL11, C-C motif chemokine 11; CLSTN2, calsyntenin 2; SNAP91, Clathrin coat assembly protein AP180; TCF24, transcription Factor 24; SMIM8, small integral membrane protein 8.

DE genes are presented. Overall, there were 144 DE mRNAs and 63 DE lncRNAs in the VC group vs. the Control group, 63 DE mRNAs and 148 DE lncRNAs in the VC + MOP group vs. the VC group and 173 DE mRNAs and 54 DE lncRNAs in the VC + MOP group vs. the Control group. The most significantly upregulated DE genes in VC group vs. the 
Table III. Total of 10 downregulated differentially expressed genes between groups by RNA-sequencing analysis.

A, VC vs. Control

\begin{tabular}{|c|c|c|c|c|}
\hline Tract ID & Gene name & Gene type & Fold-change & P-value \\
\hline ENSRNOG00000054630 & AABR07050146.1 & lincRNA & 0.48 & $4.8 \times 10^{-2}$ \\
\hline ENSRNOG00000004517 & $I G F 1$ & Protein coding & 0.49 & $1.8 \times 10^{-3}$ \\
\hline ENSRNOG00000061865 & AABR07019334.1 & lincRNA & 0.55 & $2.5 \times 10^{-2}$ \\
\hline ENSRNOG00000001300 & $P 2 R X 4$ & Protein coding & 0.58 & $2.4 \times 10^{-2}$ \\
\hline ENSRNOG00000053160 & $A A B R 07071659.2$ & lincRNA & 0.58 & $3.4 \times 10^{-2}$ \\
\hline ENSRNOG00000009734 & $A K R 1 B 10$ & Protein coding & 0.60 & $9.2 \times 10^{-3}$ \\
\hline ENSRNOG00000060166 & AABR07069067.1 & lincRNA & 0.62 & $9.9 \times 10^{-3}$ \\
\hline ENSRNOG00000020945 & $M S 4 A 1$ & Protein coding & 0.63 & $3.7 \times 10^{-2}$ \\
\hline ENSRNOG00000060865 & AABR07013167.1 & lincRNA & 0.64 & $4.4 \times 10^{-2}$ \\
\hline ENSRNOG00000056171 & $A C 107505.1$ & lincRNA & 0.64 & $5.0 \times 10^{-2}$ \\
\hline
\end{tabular}

$\mathrm{B}, \mathrm{VC}+\mathrm{MOP}$ vs. VC

\begin{tabular}{|c|c|c|c|c|}
\hline Tract ID & Gene name & Gene type & Fold-change & P-value \\
\hline ENSRNOG00000002224 & YIPF7 & Protein coding & 0.47 & $1.9 \times 10^{-2}$ \\
\hline ENSRNOG00000002207 & $G U F 1$ & Protein coding & 0.48 & $1.9 \times 10^{-2}$ \\
\hline ENSRNOG00000026976 & VOM2R57 & Protein coding & 0.52 & $4.0 \times 10^{-3}$ \\
\hline ENSRNOG00000058242 & EPS8L3 & Protein coding & 0.59 & $1.8 \times 10^{-2}$ \\
\hline ENSRNOG00000048209 & EPS8L3 & Protein coding & 0.60 & $3.0 \times 10^{-2}$ \\
\hline ENSRNOG00000059026 & $A A B R 07055885.1$ & lincRNA & 0.62 & $2.8 \times 10^{-2}$ \\
\hline ENSRNOG00000027142 & OOG1 & Protein coding & 0.63 & $6.5 \times 10^{-3}$ \\
\hline ENSRNOG00000003409 & NEWGENE_1565644 & Protein coding & 0.64 & $4.9 \times 10^{-2}$ \\
\hline ENSRNOG00000021010 & $A R L 2$ & Protein coding & 0.68 & $3.5 \times 10^{-2}$ \\
\hline ENSRNOG00000054516 & AABR07056680.1 & lincRNA & 0.68 & $4.6 \times 10^{-2}$ \\
\hline
\end{tabular}

$\mathrm{C}, \mathrm{VC}+\mathrm{MOP}$ vs. Control

\begin{tabular}{|c|c|c|c|c|}
\hline Tract ID & Gene name & Gene type & Fold-change & P-value \\
\hline ENSRNOG00000046897 & $A T R X$ & Protein coding & 0.41 & $4.0 \times 10^{-3}$ \\
\hline ENSRNOG00000008074 & CYP11A1 & Protein coding & 0.44 & $3.2 \times 10^{-2}$ \\
\hline ENSRNOG00000053160 & $A A B R 07071659.2$ & lincRNA & 0.46 & $1.1 \times 10^{-2}$ \\
\hline ENSRNOG00000004517 & $I G F 1$ & Protein coding & 0.47 & $9.5 \times 10^{-4}$ \\
\hline ENSRNOG00000002079 & MAPK10 & Protein coding & 0.51 & $3.7 \times 10^{-2}$ \\
\hline ENSRNOG00000060545 & $A C 125688.1$ & lincRNA & 0.52 & $8.0 \times 10^{-4}$ \\
\hline ENSRNOG00000036641 & LOC689065 & protein coding & 0.53 & $2.6 \times 10^{-2}$ \\
\hline ENSRNOG00000061906 & $A A B R 07024261.1$ & protein coding & 0.54 & $2.0 \times 10^{-2}$ \\
\hline ENSRNOG00000057691 & AC112557.1 & lincRNA & 0.58 & $7.7 \times 10^{-4}$ \\
\hline ENSRNOG00000009734 & AKRIB10 & protein coding & 0.58 & $1.1 \times 10^{-2}$ \\
\hline
\end{tabular}

lincRNA, long intragenic non-coding RNA; VC, varicocele; MOP, Morinda officinalis polysaccharide; IGF1, insulin-like growth factor; P2RX4, purinergic receptor P2X 4; MS4A1, membrane-spanning 4A1; YIPF7, Yip1 domain family member 7; GUF1, GUF1 homolog, GTPase; EPS8L3, epidermal growth factor receptor kinase substrate 8-like protein 3; OOG1, oogenesin 1; ARL2, ADP-ribosylation factor-like protein 2; ATRX, ATP-dependent helicase ATRX; CYP11A1, cholesterol side-chain cleavage enzyme.

Control group, VC + MOP group vs. the VC group, and VC + MOP group vs. the Control group were TMEM255B, actin g 2 smooth muscle and calsyntenin 2 , respectively. The most significantly downregulated DE genes in VC group vs. the
Control group, $\mathrm{VC}+\mathrm{MOP}$ group vs. the VC group, and VC + MOP group vs. the Control group were AABR07050146.1, YIPF7 and ATP-dependent helicase ATRX chromatin remodeler, respectively. 

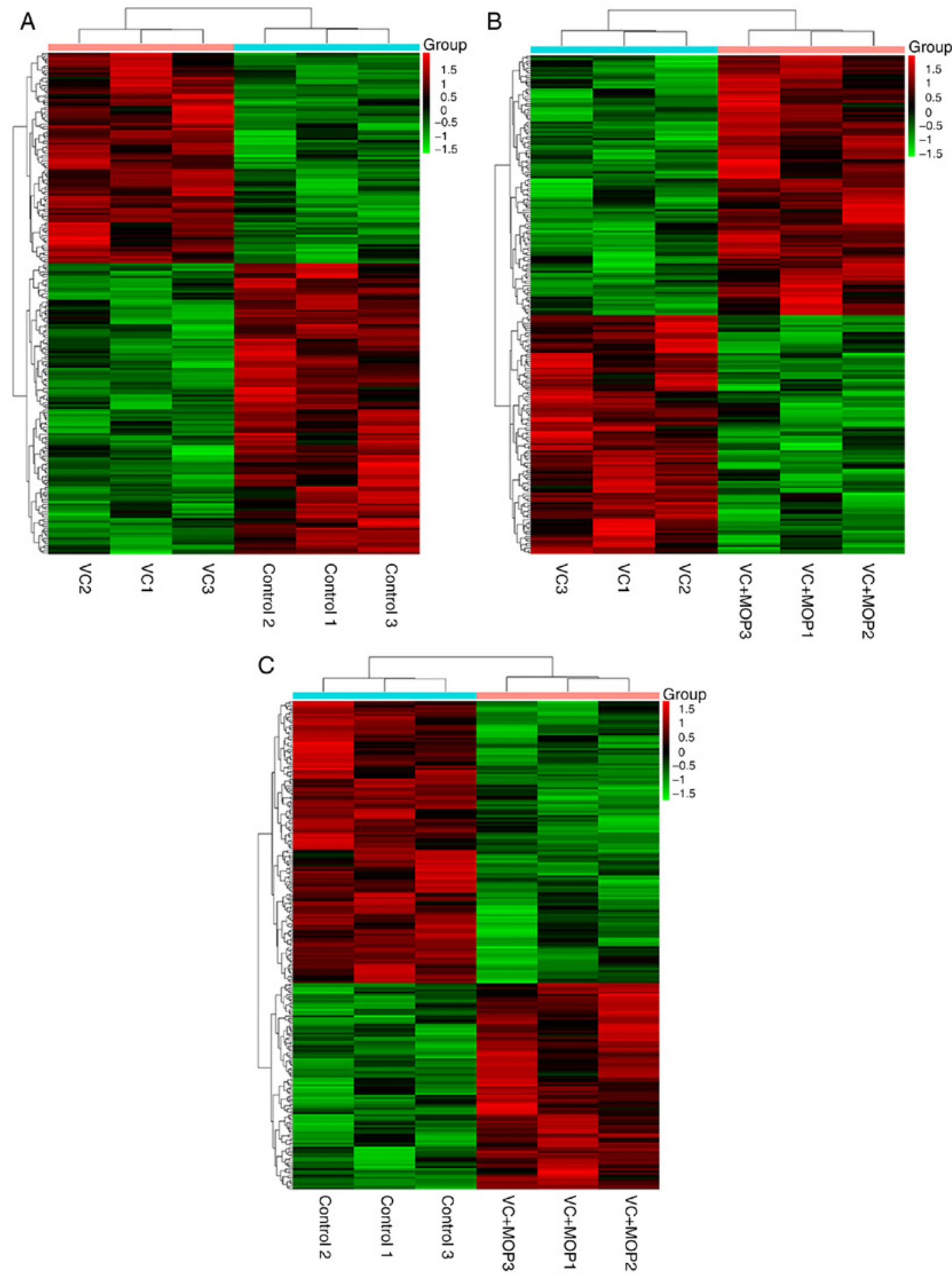

Figure 1. Hierarchical cluster analysis of differentially expressed genes between test groups. (A) VC and control, (B) VC $+\mathrm{MOP}$ and VC, and (C) VC $+\mathrm{MOP}$ and control groups. Each row represents one gene, each column represents one sample; the red and green strips represent the upregulated and downregulated differentially expressed genes, respectively. The genes with fold-change $>1.2$ and $\mathrm{P} \leq 0.05$ were selected as the differentially expressed genes. VC, varicocele; MOP, Morinda officinalis polysaccharide.

$G O$ and KEGG analyses of DE genes. To elucidate the functions of the DE genes, GO term enrichment and KEGG pathway analyses were performed. The GO functional annotations were classified into three categories: Molecular function, cellular components and biological processes. The top 10 enriched GO terms of DE genes, both upregulated and downregulated (Figs. 4 and 5) and the top 10 enriched KEGG pathways of DE genes, both upregulated and downregulated, (Fig. 6) are shown.
Co-expression network of the chosen DE mRNAs and lncRNAs. Correlation coefficients of selected DE mRNAs (protein-coding genes) and lncRNAs (non-protein-coding genes) were calculated to build a co-expression network (Pearson correlation coefficient $\geq 0.8 ; \mathrm{P} \leq 0.05$; false discovery rate, $\leq 1$; Fig. 7). These DE mRNAs and lncRNAs presented in Fig. 7 were selected according to their function based on the GO term enrichment result, but not the total DE genes. The enriched DE genes were selected based on the GO terms 

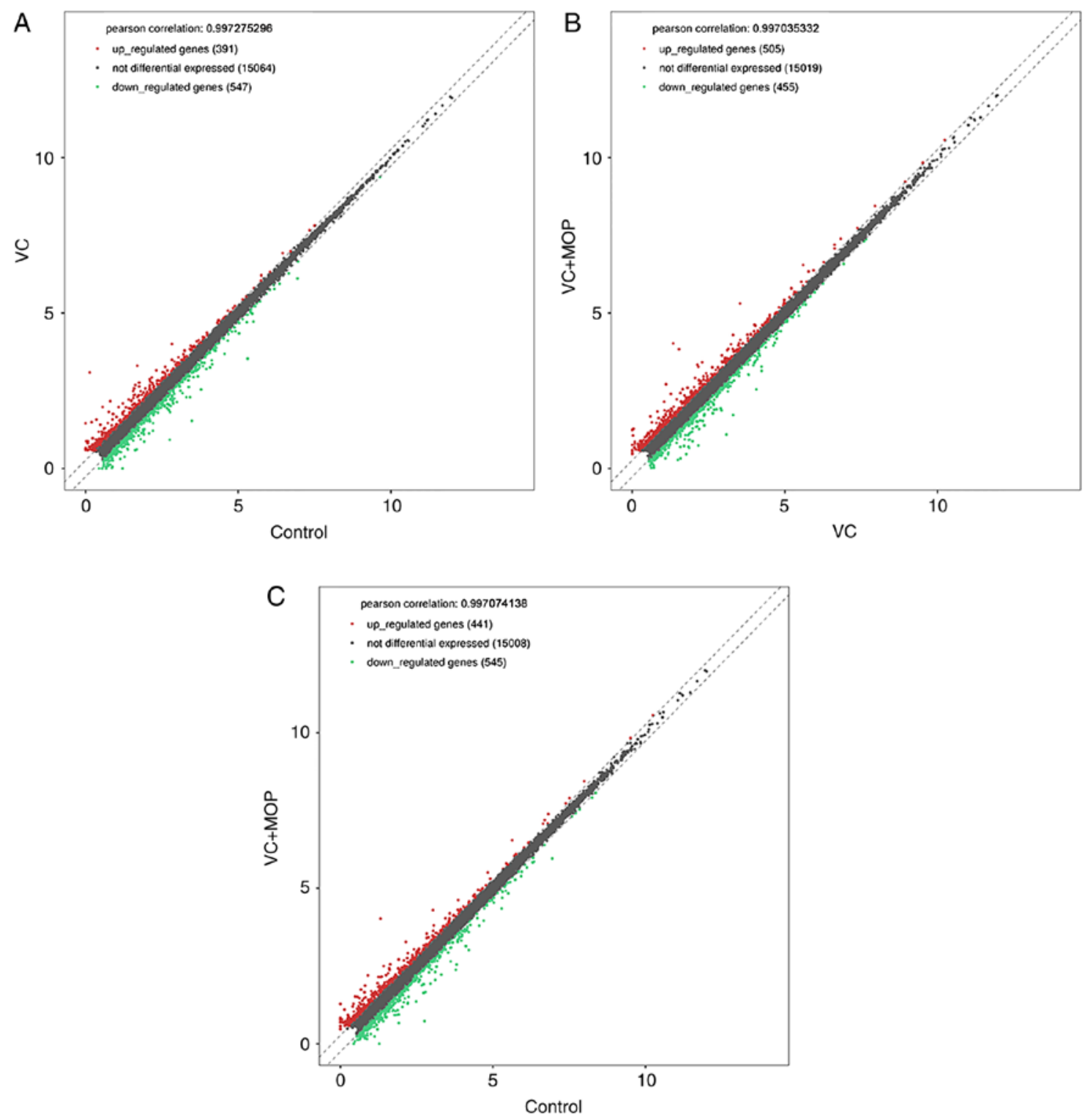

Figure 2. Scatter plots showing upregulated and downregulated differentially expressed genes. (A) VC and control, (B) VC $+\mathrm{MOP}$ and VC, and (C) VC $+\mathrm{MOP}$ and control groups. The $\mathrm{x}$-axis and $\mathrm{y}$-axis represent the average $\log 2$ (FPKM) of genes in compared groups, dotted line defines the fold-change $>1.2$. The red and green plots represent upregulated and downregulated differentially expressed genes, respectively; the gray plots represent the genes with no significant difference. VC, varicocele; MOP, Morinda officinalis polysaccharide.

the present study were interested in, such as the 'response to steroid hormone', 'oxidation-reduction process', 'positive regulation of MAPK cascade' and 'DNA repair' terms.

Validation of DE mRNAs and lncRNAs through RT-qPCR. The DE mRNAs and IncRNAs identified by RNA-Seq were validated using RT-qPCR. The results are presented in Table IV and Fig. 8. There were six DE mRNAs and four DE lncRNAs in the VC group vs. the Control group, one DE mRNA and four DE lncRNAs in the VC + MOP group vs. the VC group and four DE mRNAs and two DE lncRNAs in the $\mathrm{VC}+\mathrm{MOP}$ group vs. the Control group. Varicocele and MOP treatment appeared to have opposing effects on the expression levels of YIPF7, AABR07007833.1, AABR07014649.1 and $A A B R 07069067.1$. To be specific, the varicocele increased the expression levels of YIPF7, AABR07007833.1 and $A A B R 07014649.1$, and decreased the expression level of $A A B R 07069067.1$, while MOP treatment reversed these changes.

\section{Discussion}

In our previous study, varicocele induced reproductive dysfunction in male rats via mechanisms such as destruction of the seminiferous epithelium and TJ structure, downregulation of TJ proteins (occludin, claudin-11 and zona occludens protein 1), deregulation of hormone levels and an increase in cytokine (TGF- $\beta 3$ and TNF- $\alpha$ ) levels. MOP repaired varicocele-induced damage and promoted spermatogenesis (the most effective dose was found to be $300 \mathrm{mg} / \mathrm{kg}$ ) (17). However, the molecular mechanisms underlying the pathophysiology of varicocele and the therapeutic effect of MOP are still unknown. In the present study, mRNA and lncRNA sequencing analyses, combined with validation 

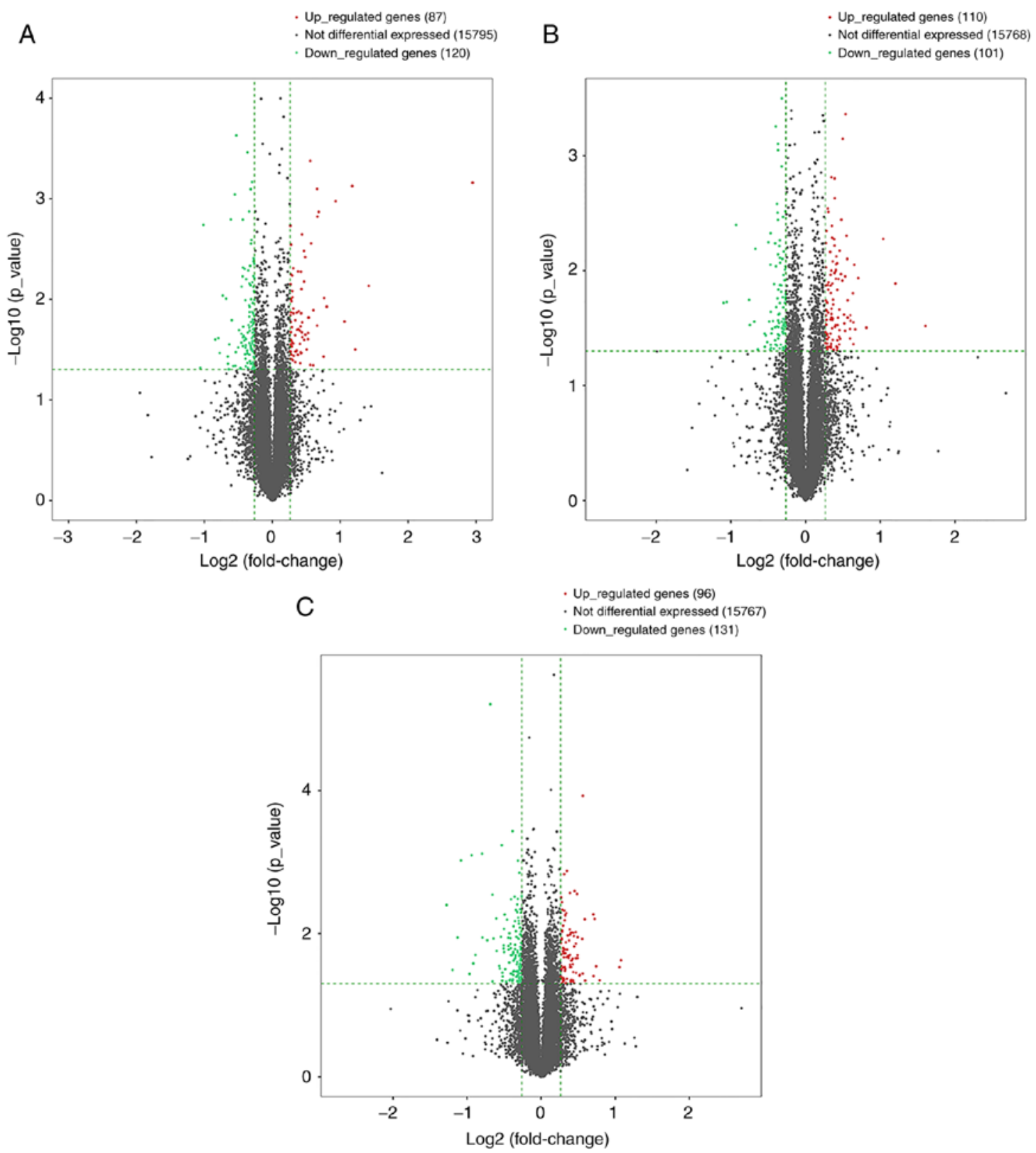

Figure 3. Volcano plots of differentially expressed genes. (A) VC and control, (B) VC + MOP and VC and (C) VC + MOP and control groups. The X-axis and the Y-axis represent the $\log 2$ (fold-change) and - $\log 10$ (P-value), respectively; two vertical dotted lines define the fold-change $>1.2$, one horizontal dotted line defines the $\mathrm{P} \leq 0.05$. The red and green plots represent upregulated and downregulated differentially expressed genes, respectively; the gray plots represent the genes with no significant difference. The genes with fold-change $>1.2$ and $\mathrm{P} \leq 0.05$ were selected as the differentially expressed genes. VC, varicocele; MOP, Morinda officinalis polysaccharide.

of DE genes through RT-qPCR, were performed to bridge these data gaps.

According to GO results, the pathophysiology of varicocele may be associated with 'epidermal growth factor receptor binding', 'ligand-gated calcium channel activity', 'growth factor receptor binding', 'histone kinase activity' and 'microtubule motor activity'. Notably, numerous DE genes between the VC and control groups were enriched in cancer-related pathways, such as the 'p53 signaling pathway', which is downregulated in breast cancer and melanoma; this warrants further research and validation.

According to the KEGG pathway analysis, "cytokinecytokine receptor interaction', 'Wnt signaling pathway' and 'p53 signaling pathway' were all implicated in the varicocele-therapeutic effect of MOP. In our previous study, the levels of TGF- $\beta 3$ and TNF- $\alpha$ were upregulated in experimental rat left testicular tissue and downregulated following MOP treatment, potentially through the cytokine-cytokine receptor interaction pathway (17). The Wnt signaling pathway plays a notable role in the normal physiology and pathology of the male reproductive system. The $\mathrm{Wnt} / \beta$-catenin pathway is involved in the annexin 5-mediated stimulation of testosterone synthesis (35), and its dysregulation is linked to the development of granulosa cell tumors in the testis (36). Additionally, the activation of Wnt/ $/$-catenin signaling in Sertoli cells results in germ cell loss and seminiferous tubule degeneration (37). Moreover, the Wnt/ $\beta$-catenin signaling pathway facilitates the proliferation of spermatogonial stem cells in the testis (38). 

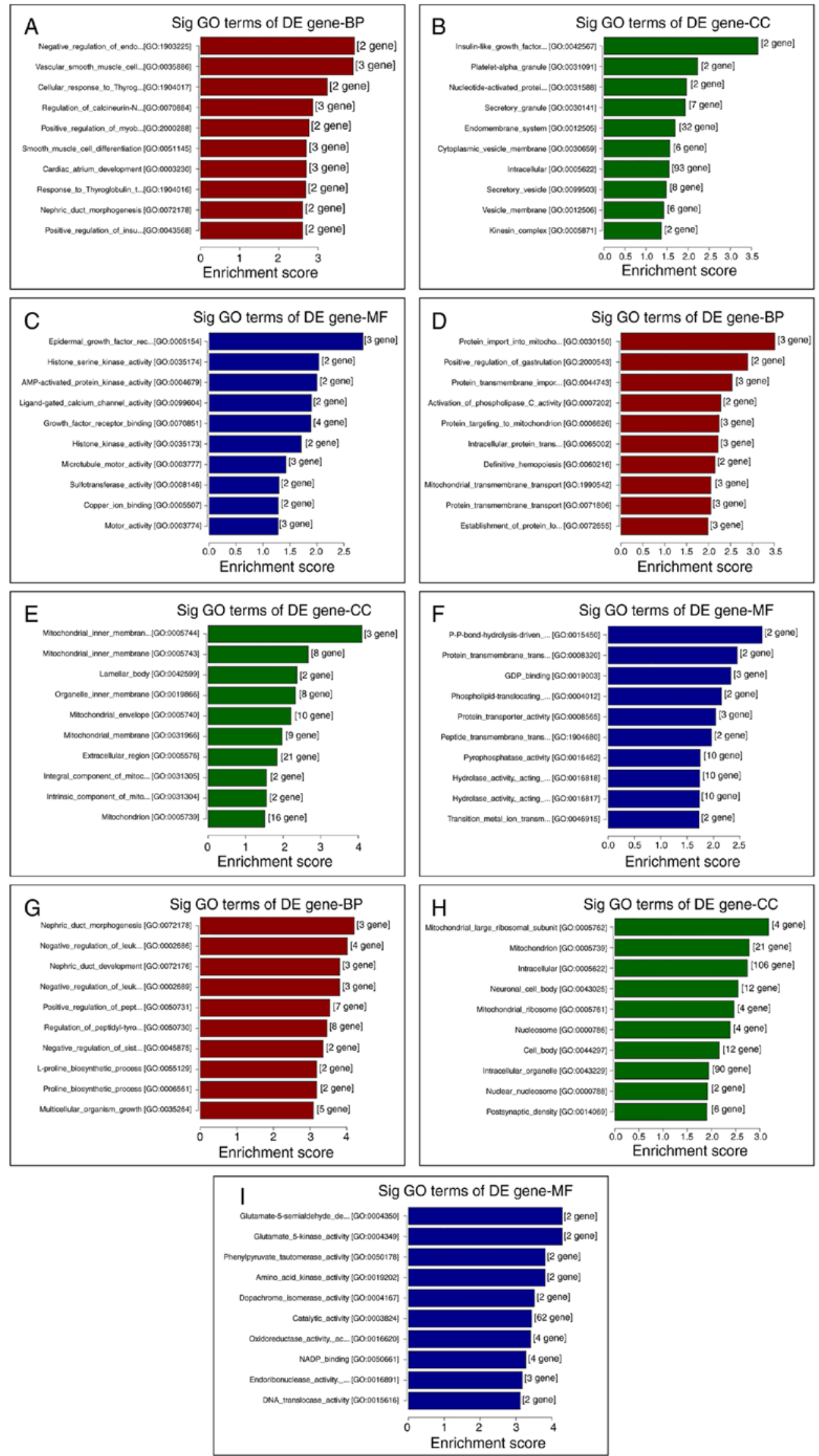

Figure 4. GO analysis of the top $10 \mathrm{DE}$ genes between different groups, as ranked by their enrichment score. Top 10 (A) BP, (B) CC and (C) MF terms between the VC and control groups. Top 10 (D) BP, (E) CC and (F) MF terms between the VC+ MOP and VC groups. Top 10 (G) BP, (H) CC and (I) MF terms between the $\mathrm{VC}+\mathrm{MOP}$ and control groups. DE genes with fold-change $>1.2$ and $\mathrm{P} \leq 0.05$ were used. BP, biological process; CC, cellular component; MF, molecular function; GO, Gene Ontology; DE, differentially expressed; Sig, significant; VC, varicocele; MOP, Morinda officinalis polysaccharide. 

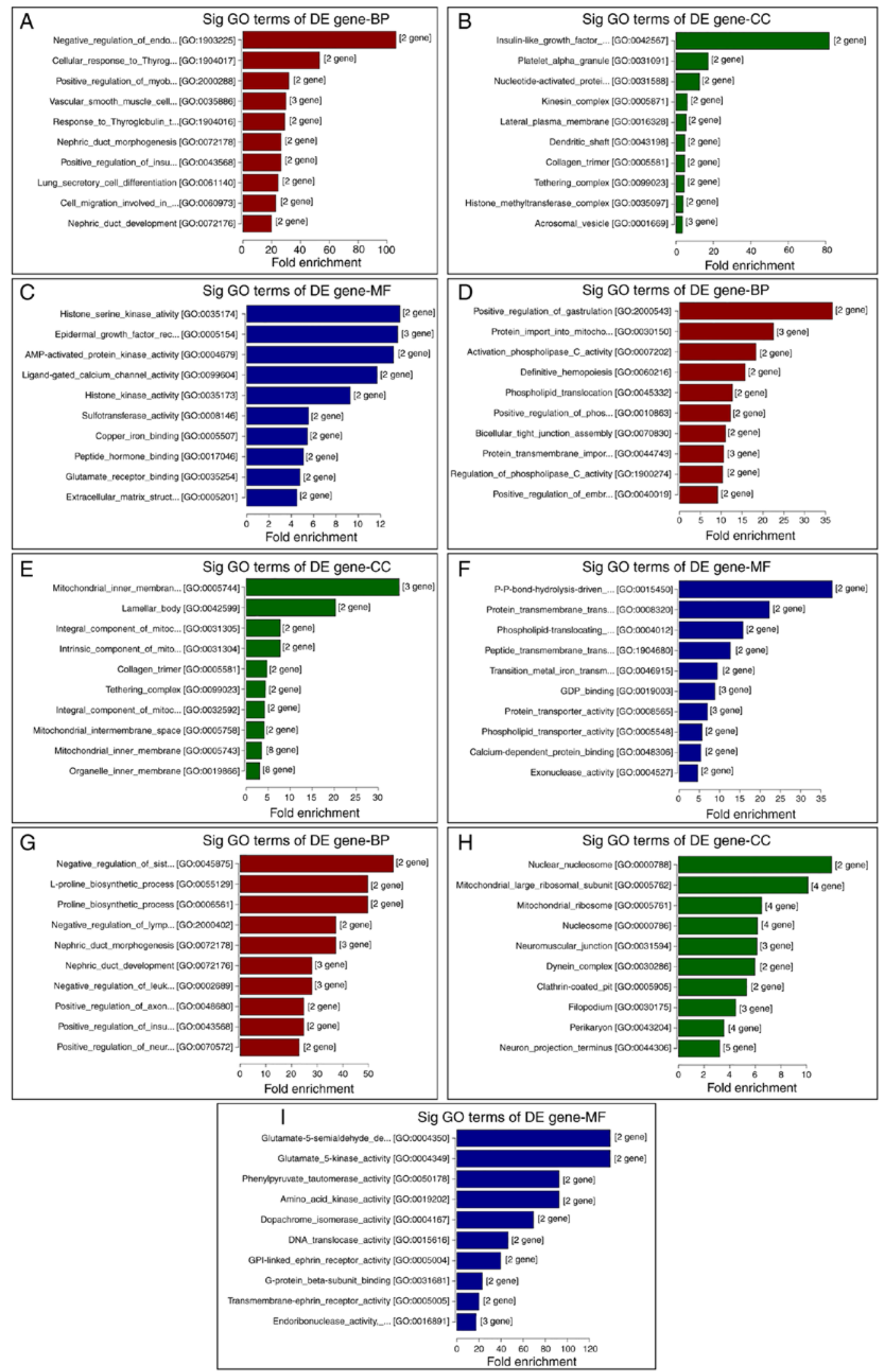

Figure 5. GO analysis of the top $10 \mathrm{DE}$ genes between different groups, as ranked by fold-enrichment. Top 10 (A) BP, (B) CC and (C) MF between the VC and control groups. Top 10 (D) BP, (E) CC and (F) MF terms between the VC + MOP and VC groups. Top 10 (G) BP, (H) CC and (I) MF terms, respectively, between the $\mathrm{VC}+\mathrm{MOP}$ and control groups. DE genes with fold-change $>1.2$ and $\mathrm{P} \leq 0.05$ were used. BP, biological process; CC, cellular component; MF, molecular function; GO, Gene Ontology; DE, differentially expressed; Sig, significant; VC, varicocele; MOP, Morinda officinalis polysaccharide.

Thus, proper regulation of $\mathrm{Wnt} / \beta$-catenin signaling is necessary for adult spermatogenesis, and its disruption may result in infertility (39). The results of the present study provide compelling evidence for the involvement of $\mathrm{Wnt} / \beta$-catenin signaling 
A

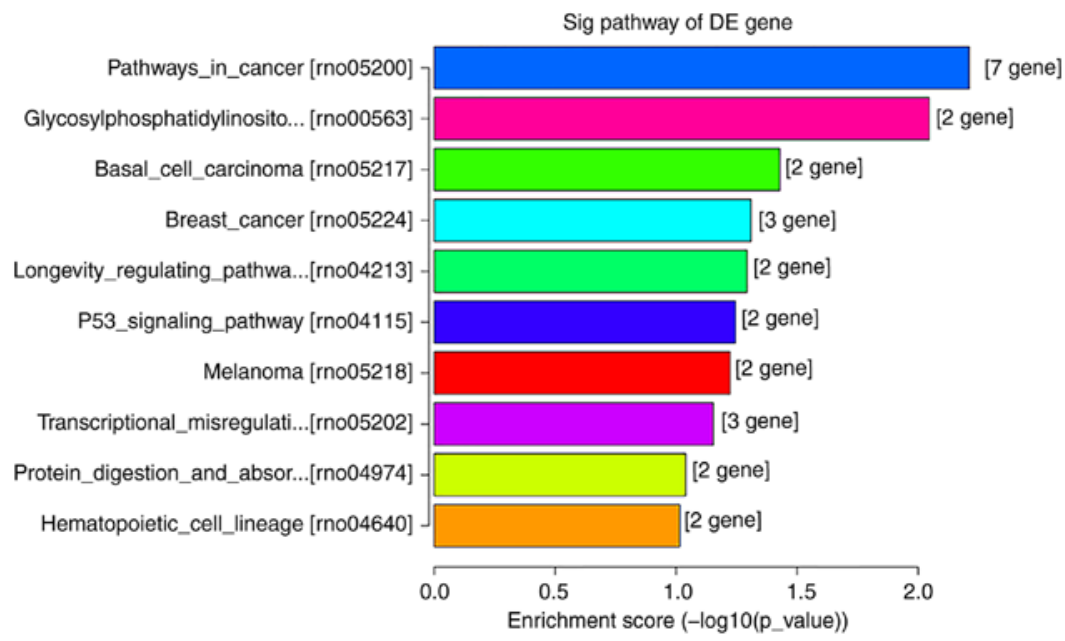

B

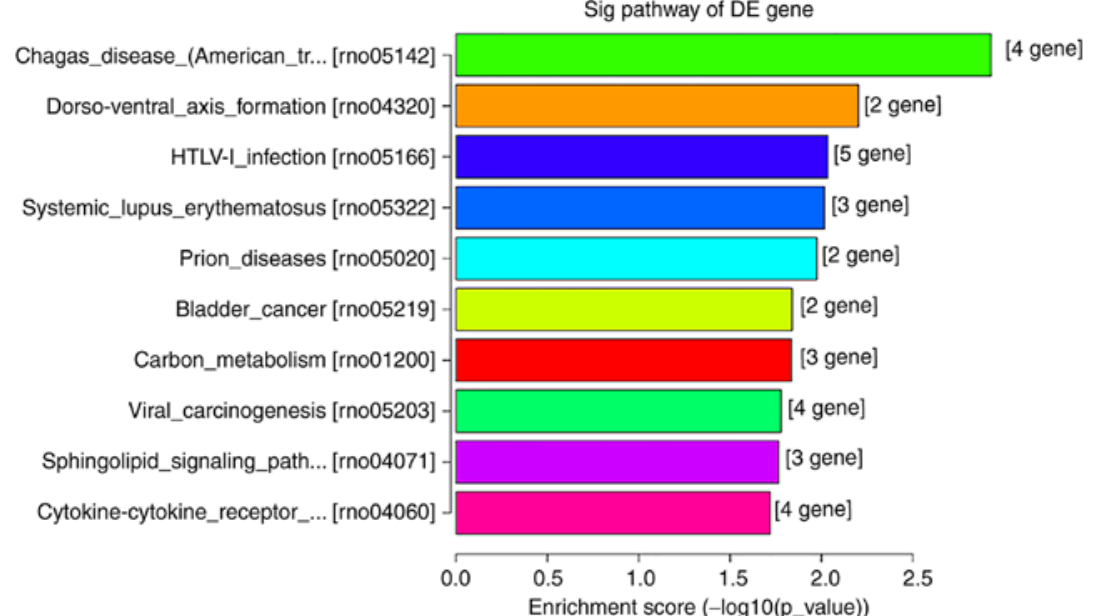

C

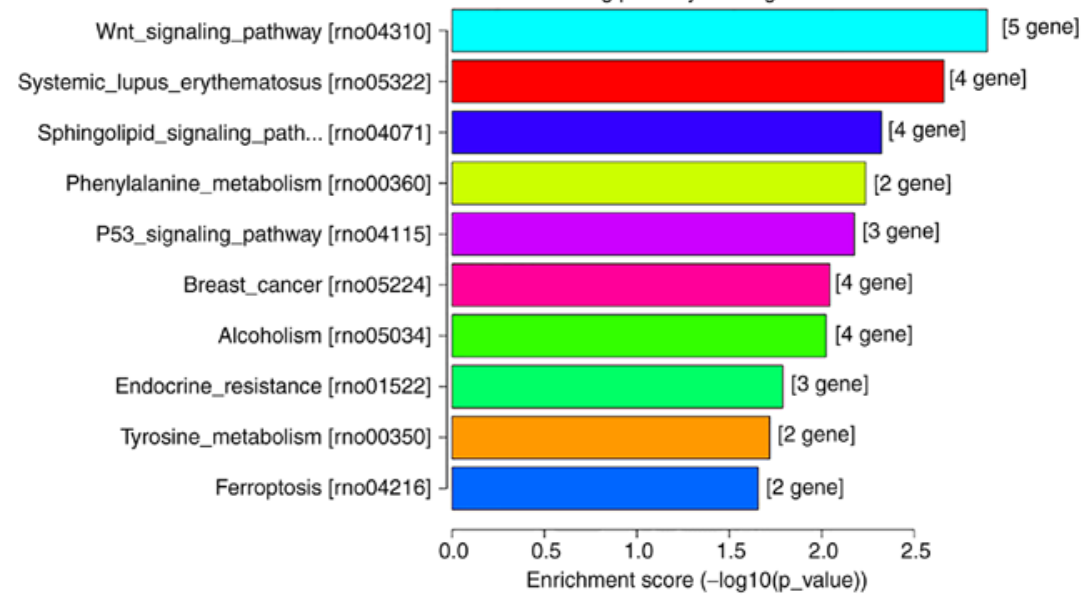

Figure 6. Kyoto Encyclopedia of Genes and Genomes analysis of pathways corresponding to the top 10 differentially expressed genes between groups. (A) VC and control groups, (B) the VC + MOP and VC groups, and (C) the VC + MOP and control groups. Differentially expressed genes with fold-change $>1.2$ and $\mathrm{P} \leq 0.05$ were used. VC, varicocele; MOP, Morinda officinalis polysaccharide; DE, differentially expressed; Sig, significant.

in varicocele progression and the therapeutic effect of MOP in varicocele; this evidence is worthy of further research.

The RT-qPCR analysis revealed that one coding gene, $Y I P F 7$, and three non-coding genes, AABR07007833.1, $A A B R 07014649.1$ and $A A B R 07069067.1$, were DE between the $\mathrm{VC}$ and Control groups, and between the $\mathrm{VC}+\mathrm{MOP}$ and $\mathrm{VC}$ groups. Varicocele and MOP treatment appeared to have opposing effects on the expression of these genes, implying that these genes may play a role in varicocele pathophysiology and the repair effect of MOP. Specifically, varicocele increased the expression levels of YIPF7, AABR07007833.1 and $A A B R 07014649.1$, and decreased the expression level of AABR07069067.1, while MOP treatment reversed these effects. The YIP1 family of proteins are involved in protein transport between the endoplasmic reticulum and Golgi apparatus (40), as well as the regulation of membrane dynamics (41). Decreasing the expression of YIPF7 enhances the intestinal inflammatory response and upregulates the $T N F$ mRNA 
A

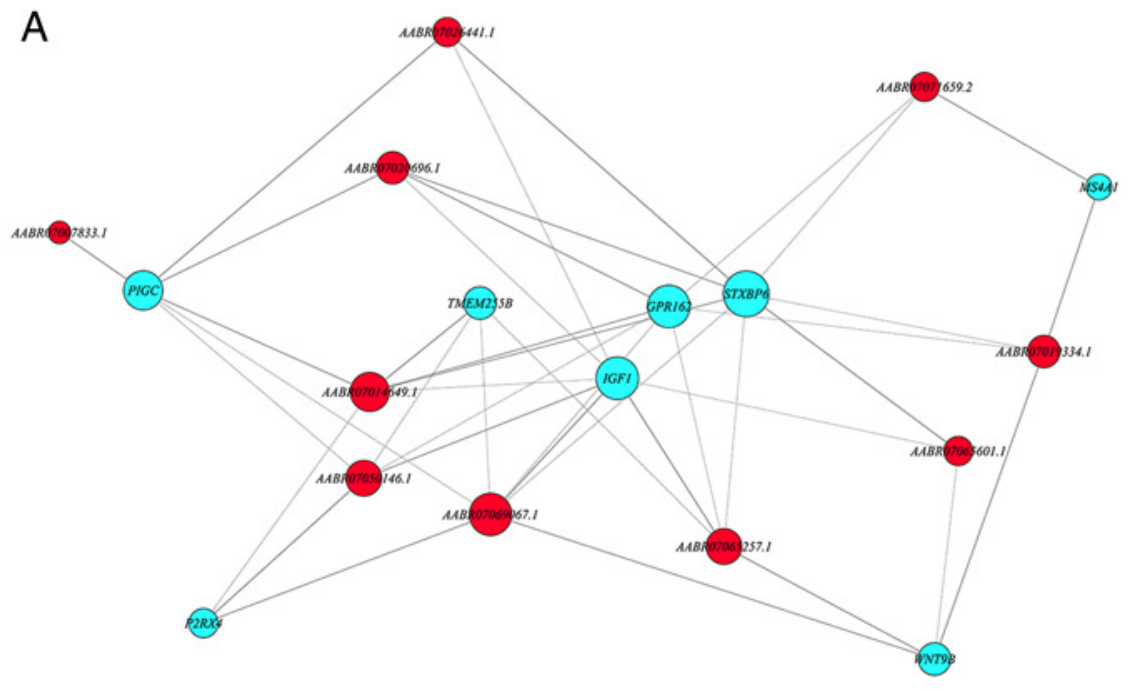

C
B

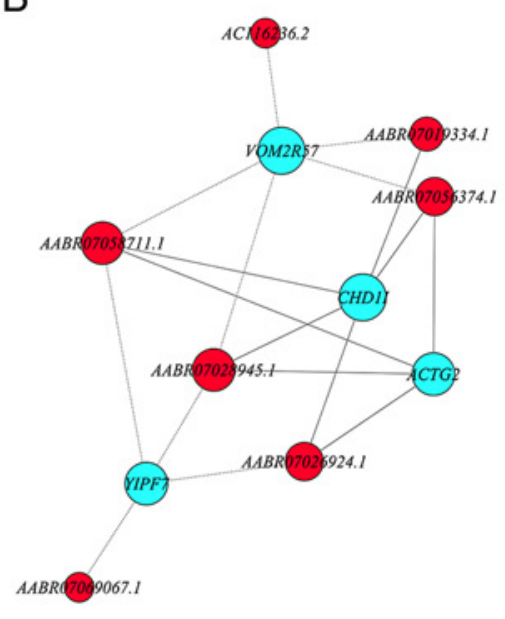

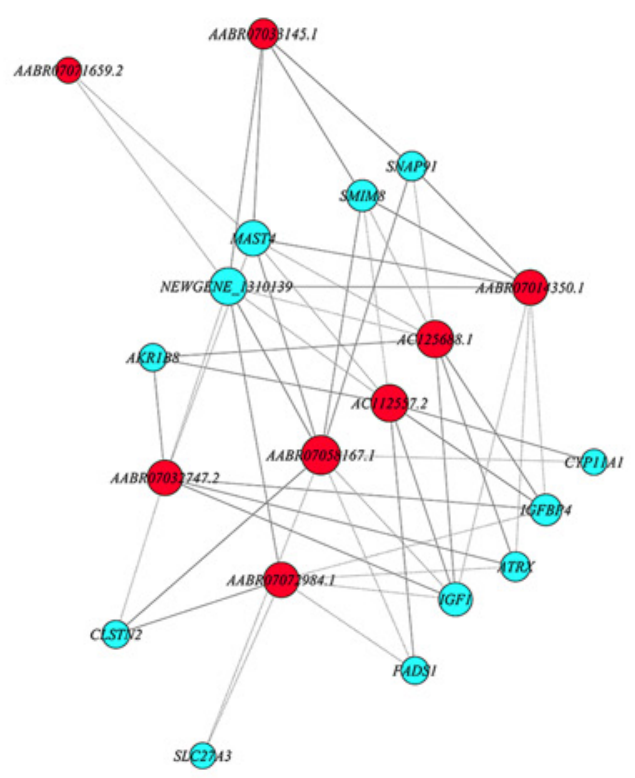

Figure 7. Co-expression network of chosen differentially expressed mRNAs and lncRNAs between different groups. (A) VC and control groups, (B) the VC + MOP and VC groups, and (C) the VC + MOP and control groups. Red and blue labels denote lncRNAs (non-protein-coding genes) and mRNAs (protein-coding genes), respectively. Solid lines and dotted lines represent positive and negative associations, respectively. IncRNAs, long non-coding RNA; VC, varicocele; MOP, Morinda officinalis polysaccharide.

level (42). The change in YIPF7 expression may be associated with the testicular inflammatory response and the fluctuation of the TNF- $\alpha$ expression level induced by varicocele and MOP. This finding is in accordance with the results of our previous study; thus, we hypothesize that YIPF7 is involved in the process of varicocele development by increasing the TNF- $\alpha$ level in the left testicular tissue, which MOP treatment is able to decrease (17). Due to the lack of extensive research on IncRNA, the functions of $A A B R 07007833.1$, $A A B R 07014649.1$ and $A A B R 07069067.1$ remain elusive. In the present study, according to the co-expression network analysis, these non-coding genes were positively associated with phosphatidylinositol glycan anchor biosynthesis class $\mathrm{C}$ insulin-like growth factor 1 (IGF1), P2RX4, TMEM255B and $W N T 9 B$, as well as others.

Aldo-keto reductase family 1 , member B8 (AKRlB8), leukocyte immunoglobulin-like receptor B2 (NILR1), P2RX4,
TMEM $255 B$ and $W N T 9 B$ were also DE between the VC and control groups. TMEM255B, also known as FAM70B, encodes the transmembrane protein $255 \mathrm{~b}$, which has been proposed as a prognostic marker for muscle-invasive bladder cancer (43). The expression level of TMEM255B in the VC group was $\sim 63$-fold higher compared with that of the control group. Such a large difference in expression levels suggests that TMEM255B could be used as a novel diagnostic maker for varicocele; however, further clinical research is required to confirm this hypothesis. WNT9B takes part in the Wnt/ $\beta$-catenin signaling pathway, described as aforementioned. $P 2 R X 4$, also known as $P 2 X 4$ or $P 2 X 4 R$, encodes the ATP-gated P2RX4 ion channel. According to a previous study, extracellular ATP is a danger molecule for peritubular cells and aggravates inflammatory responses in the testis (44). P2RX4 is involved in pain processing (45-47), and its downregulation has been shown to moderate allergen-induced airway inflammation (48). 
Table IV. Expression of differentially expressed genes by reverse transcription-quantitative PCR.

A, VC vs. Control

\begin{tabular}{llcr}
\hline Gene name & Gene type & Mean fold-change \pm SD & P-value \\
\hline AKR1B8 & Protein coding & $0.47 \pm 1.1 \times 10^{-2}$ & 0.024 \\
NILR1 & Protein coding & $2.98 \pm 6.2 \times 10^{-4}$ & 0.044 \\
P2RX4 & Protein coding & $0.48 \pm 4.1 \times 10^{-2}$ & 0.041 \\
TMEM255B & Protein coding & $63.34 \pm 1.4 \times 10^{-2}$ & 0.044 \\
WNT9B & Protein coding & $0.61 \pm 1.0 \times 10^{-3}$ & 0.026 \\
YIPF7 & Protein coding & $2.43 \pm 6.3 \times 10^{-3}$ & 0.031 \\
AABR07007833.1 & lincRNA & $1.51 \pm 2.6 \times 10^{-5}$ & 0.033 \\
AABR07014649.1 & lincRNA & $1.42 \pm 1.5 \times 10^{-3}$ & 0.025 \\
AABR07050146.1 & lincRNA & $0.81 \pm 5.1 \times 10^{-5}$ & 0.035 \\
AABR07069067.1 & lincRNA & $0.47 \pm 6.5 \times 10^{-4}$ & 0.014 \\
\hline
\end{tabular}

$\mathrm{B}, \mathrm{VC}+\mathrm{MOP}$ vs. VC

\begin{tabular}{llcc}
\hline Gene name & Gene type & Mean fold-change \pm SD & P-value \\
\hline YIPF7 & Protein coding & $0.31 \pm 7.3 \times 10^{-3}$ & 0.025 \\
AABR07007833.1 & lincRNA & $0.44 \pm 3.9 \times 10^{-5}$ & 0.0092 \\
$A A B R 07014649.1$ & lincRNA & $0.77 \pm 1.0 \times 10^{-3}$ & 0.0011 \\
$A A B R 07069067.1$ & lincRNA & $2.27 \pm 7.3 \times 10^{-4}$ & 0.0047 \\
$A A B R 07004428.1$ & lincRNA & $0.52 \pm 1.4 \times 10^{-3}$ & 0.049 \\
\hline
\end{tabular}

$\mathrm{C}, \mathrm{VC}+\mathrm{MOP}$ vs. Control

\begin{tabular}{llcl}
\hline Gene name & Gene type & Mean fold-change \pm SD & P-value \\
\hline$A K R 1 B 8$ & Protein coding & $0.50 \pm 9.4 \times 10^{-3}$ & 0.0072 \\
$C L S T N 2$ & Protein coding & $7.82 \pm 1.5 \times 10^{-3}$ & 0.023 \\
GPR162 & Protein coding & $1.49 \pm 2.0 \times 10^{-4}$ & 0.038 \\
WNT9B & Protein coding & $0.58 \pm 1.1 \times 10^{-3}$ & 0.030 \\
$A C 125688.1$ & lincRNA & $0.54 \pm 3.0 \times 10^{-2}$ & 0.0022 \\
$A A B R 07058711.1$ & lincRNA & $1.22 \pm 2.2 \times 10^{-4}$ & 0.040
\end{tabular}

lincRNA, long intragenic non-coding RNA; VC, varicocele; MOP, Morinda officinalis polysaccharide; AKR1B8, Aldo-keto reductase family 1, member B8; CLSTN2, calsyntenin 2; GPR162, G protein-coupled receptor 162; NILR1, leukocyte immunoglobulin like receptor B2; P2RX4, purinergic receptor P2X 4; TMEM255B, transmembrane protein 225B; WNT9B, Wnt family member 9B; YIPF7, Yip1 domain family member 7 .

Although the function of P2RX4 in the male reproductive system is unclear, according to its diverse biological activities under physiological and pathological conditions (44-49), it is hypothesized that changes in P2RX4 expression levels are involved in varicocele pathogenesis via the stimulation of inflammatory responses and disruption of transmembrane ion channel function.

In the present study, RNA sequencing and RT-qPCR were performed on rat testicular tissue to reveal the molecular mechanisms underlying varicocele pathophysiology and the restorative effect of MOP on varicocele-induced damage in male reproductive systems. The results of GO and KEGG pathway enrichment analyses showed that 'ligand-gated calcium channel activity', 'cytokine-cytokine receptor interaction' and the 'Wnt signaling pathway' may all be implicated to underlie this effect. RT-qPCR confirmed that YIPF 7 is upregulated in varicocele and downregulated by MOP. Based on its diverse biological activities and intimate connection to TNF- $\alpha$, YIPF 7 is considered a key mediator of varicocele pathogenesis and therapeutic effects of MOP. Furthermore, differential expression of $A K R 1 B 8, N I L R 1$, $P 2 R X 4, T M E M 255 B$ and $W N T 9 B$ was detected between the VC group and the control group. TMEM255B may be a potential novel diagnostic marker for varicocele; the role of $W N T 9 B$ and $P 2 R X 4$ in varicocele is possibly mediated by the activation of Wnt signaling and the regulation of transmembrane ion channels and the inflammatory response, respectively. 

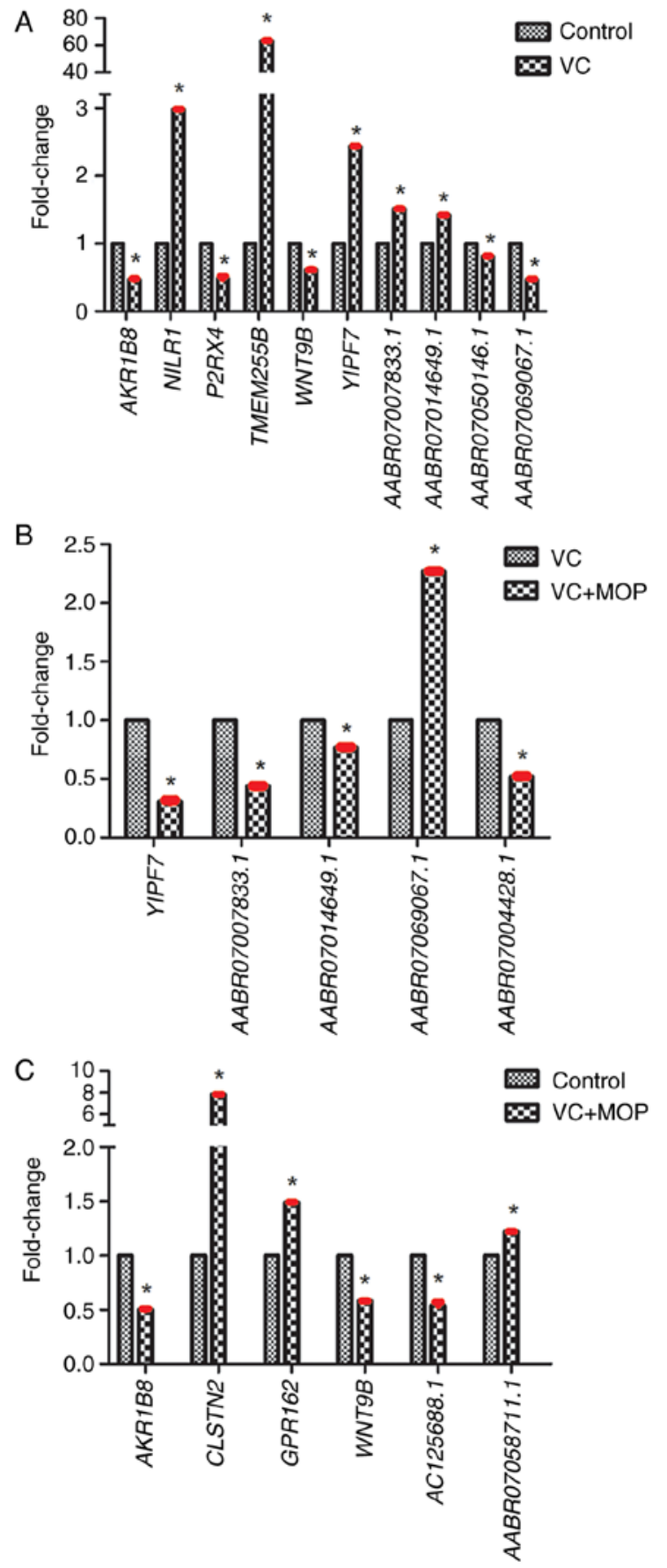

Figure 8. Expression levels of DE genes assessed by reverse transcriptionquantitative PCR. (A) DE genes between the VC and control groups. (B) DE genes between the VC + MOP and VC groups. (C) DE genes between the VC $+\mathrm{MOP}$ and control groups. ${ }^{*} \mathrm{P} \leq 0.05$ vs. respective control groups. All data are presented as mean $\pm \mathrm{SD}$, bars indicate $\mathrm{SD}$ and are shown in red, $\mathrm{n}=3$. $\mathrm{VC}$, varicocele; MOP, Morinda officinalis polysaccharide; DE, differentially expressed; AKR1B8, Aldo-keto reductase family 1, member B8; CLSTN2, calsyntenin 2; GPR162, G protein-coupled receptor 162; NILR1, leukocyte immunoglobulin like receptor $\mathrm{B} 2$; P2RX4, purinergic receptor P2X 4; TMEM255B, transmembrane protein 225B; WNT9B, Wnt family member 9B; YIPF7, Yip1 domain family member 7.

In summary, the present study provides a foundation for understanding the molecular basis of varicocele pathophysiology and the varicocele-therapeutic effect of MOP, offers insights into novel strategies for varicocele diagnosis and treatment, and suggests directions for further study.

\section{Acknowledgements}

Not applicable.

\section{Funding}

The present study was supported by The Training Program Foundation for the Talents of Health and Family Planning Commission in Fujian Province (grant no. 2018-1-74), The Start-up Foundation of Fujian Medical University (grant no. 2017XQ1005), The Research Foundation for High-Level Talents of Fujian Medical University (grant. no. XRCZX2017033), The Natural Science Foundation of Fujian Province (grant no. 2017J01819) and The Co-construction Science Foundation of National Health and Family Planning Commission-Joint Program for Tackling Key Problems of Health and Education in Fujian Province (grant no. WKJ2016-2-35).

\section{Availability of data and materials}

The datasets used and/or analyzed during the current study are available from the corresponding authors upon reasonable request. The datasets generated and analyzed during the current study are available in the Gene Expression Omnibus repository, https://www.ncbi.nlm.nih.gov/geo/query/acc.cgi (accession no. GSE139447; secure token: sfaxocskhpoxnix).

\section{Authors' contributions}

LZ performed the experiments and the statistical analysis, and drafted the manuscript. WW and $\mathrm{XZ}$ conceived the study and participated in its design and coordination. All authors read and approved the final manuscript.

\section{Ethics approval and consent to participate}

The study was approved by The Animal ApprovalCommittee of Fujian Medical University (approval no. SYXK-2012-0001).

\section{Patient consent for publication}

Not applicable.

\section{Competing interests}

The authors declare that they have no competing interests.

\section{References}

1. Leslie SW, Sajjad H and Siref LE: Varicocele. In: StatPearls. StatPearls Publishing, Treasure Island, FL, 2021.

2. Wang NN, Dallas K, Li S, Baker L and Eisenberg ML: The association between varicocoeles and vascular disease: An analysis of U.S. Claims data. Andrology 6: 99-103, 2018.

3. Reesink DJ, Huisman PM, Wiltink J, Kruger AE and Lock TMT: Sneeze and pop: A ruptured varicocele; analysis of literature, guided by a well-documented case-report. BMC Urol 19: 14, 2019.

4. Sigalos JT and Pastuszak AW: Chronic orchialgia: Epidemiology, diagnosis and evaluation. Transl Androl Urol 6 (Suppl 1): S37-S43, 2017. 
5. Afshar A, Aliaghaei A, Nazarian H, Abbaszadeh HA, Naserzadeh $P$ Fathabadi FF, Abdi S, Raee P, Aghajanpour F, Norouzian M and Abdollahifar MA: Curcumin-loaded iron particle improvement of spermatogenesis in azoospermic mouse induced by long-term scrotal hyperthermia. Reprod Sci 28: 371-380, 2021.

6. Jarow JP: Effects of varicocele on male fertility. Hum Reprod Update 7: 59-64, 2001.

7. Mehta A and Goldstein M: Microsurgical varicocelectomy: A review. Asian J Androl 15: 56-60, 2013.

8. Ahlberg NE, Bartley O and Chidekel N: Right and left gonadal veins. An anatomical and statistical study. Acta Radiol Diagn (Stockh) 4: 593-601, 1966.

9. Bach PV, Najari BB and Goldstein M: Varicocele-a case for early intervention. F1000Res 5: 1792, 2016.

10. Chiba K, Ramasamy R, Lamb DJ and Lipshultz LI: The varicocele: Diagnostic dilemmas, therapeutic challenges and future perspectives. Asian J Androl 18: 276-281, 2016.

11. Fretz PC and Sandlow JI: Varicocele: Current concepts in pathophysiology, diagnosis, and treatment. Urol Clin North Am 29: 921-937, 2002

12. Luo DY, Yang G, Liu JJ, Yang YR and Dong Q: Effects of varicocele on testosterone, apoptosis and expression of StAR mRNA in rat Leydig cells. Asian J Androl 13: 287-291, 2011.

13. Saypol DC, Howards SS, Turner TT and Miller ED Jr: Influence of surgically induced varicocele on testicular blood flow, temperature, and histology in adult rats and dogs. J Clin Invest 68 39-45, 1981.

14. Pastuszak AW and Wang R: Varicocele and testicular function. Asian J Androl 17: 659-667, 2015

15. Ozbek E, Yurekli M, Soylu A, Davarci M and Balbay MD: The role of adrenomedullin in varicocele and impotence. BJU Int 86 : 694-698, 2000

16. Kim IT, Park HJ, Nam JH, Park YM, Won JH, Choi J, Choe BK and Lee KT: In-vitro and in-vivo anti-inflammatory and antinociceptive effects of the methanol extract of the roots of Morinda officinalis. J Pharm Pharmacol 57: 607-615, 2005.

17. Zhang L, Zhao X, Wang F, Lin Q and Wang W: Effects of morinda officinalis polysaccharide on experimental varicocele rats. Evid Based Complement Alternat Med 2016: 5365291, 2016.

18. Marinov GK: On the design and prospects of direct RNA sequencing. Brief Funct Genomics 16: 326-335, 2017.

19. Li X, Wu Z, Fu X and Han W: IncRNAs: Insights into their function and mechanics in underlying disorders. Mutat Res Rev Mutat Res 762: 1-21, 2014.

20. Kazimierczyk M,KasprowiczMK, Kasprzyk MEand Wrzesinski J: Human long noncoding RNA interactome: Detection, characterization and function. Int J Mol Sci 21: 1027, 2020

21. Li L, Zhuang Y, Zhao X and Li X: Long non-coding RNA in neuronal development and neurological disorders. Front Genet 9: $744,2018$.

22. Hu Y, Lin J, Fang H, Fang J, Li C, Chen W, Liu S, Ondrejka S, Gong Z, Reu F, et al: Targeting the MALAT1/ PARP1/LIG3 complex induces DNA damage and apoptosis in multiple myeloma. Leukemia 32: 2250-2262, 2018

23. Mirza AH, Kaur S and Pociot F: Long non-coding RNAs as novel players in $\beta$ cell function and type 1 diabetes. Hum Genomics 11: $17,2017$.

24. Zhang Y, Hou YM, Gao F, Xiao JW, Li CC and Tang Y: lncRNA GAS5 regulates myocardial infarction by targeting the miR-525-5p/CALM2 axis. J Cell Biochem 120: 18678-18688, 2019.

25. Morlando M and Fatica A: Alteration of epigenetic regulation by long noncoding RNAs in cancer. Int J Mol Sci 19: 570, 2018.

26. Yang Y, Junjie P, Sanjun C and Ma Y: Long non-coding RNAs in colorectal cancer: Progression and future directions. J Cancer 8 : 3212-3225, 2017.

27. Loewen G, Jayawickramarajah J, Zhuo Y and Shan B: Functions of lncRNA HOTAIR in lung cancer. J Hematol Oncol 7: 90, 2014

28. Wang X, Ruan Y, Wang X, Zhao W, Jiang Q, Jiang C, Zhao Y, $\mathrm{Xu}$ Y, Sun F, Zhu Y, et al: Long intragenic non-coding RNA lincRNA-p21 suppresses development of human prostate cancer. Cell Prolif 50: e12318, 2017.

29. Dong H, Zhang Q, Li Y, Li L, Lan W, He J, Li H, Xiong Y and Qin W: Extraction, characterization and antioxidant activities of polysaccharides of Chuanminshen violaceum. Int J Biol Macromol 86: 224-232, 2016.
30. Xu SY, Liu JP, Huang X, Du LP, Shi FL, Dong R, Huang XT, Zheng K, Liu Y and Cheong KL: Ultrasonic-microwave assisted extraction, characterization and biological activity of pectin from jackfruit peel. Lwt 90: 577-582, 2018.

31. Turner TT: The study of varicocele through the use of animal models. Hum Reprod Update 7: 78-84, 2001

32. Pertea M, Kim D, Pertea GM, Leek JT and Salzberg SL: Transcript-level expression analysis of RNA-seq experiments with HISAT, StringTie and Ballgown. Nat Protoc 11: 1650-1667, 2016.

33. Kim D, Langmead B and Salzberg SL: HISAT: A fast spliced aligner with low memory requirements. Nat Methods 12: 357-360, 2015.

34. Livak KJ and Schmittgen TD: Analysis of relative gene expression data using real-time quantitative PCR and the 2(-Delta Delta C(T)) method. Methods 25: 402-408, 2001.

35. Zhang T, Zheng T, Wang C, Zhang W, Jia D, Wang R and Qiao B: Effects of Wnt/3-catenin signaling pathway and star D7 on testosterone synthesis. Acta Endocrinol (Buchar) 14: 155-162, 2018.

36. Boyer A, Paquet M, Lague MN, Hermo L and Boerboom D Dysregulation of WNT/CTNNB1 and PI3K/AKT signaling in testicular stromal cells causes granulosa cell tumor of the testis. Carcinogenesis 30: 869-878, 2009

37. Boyer A, Hermo L, Paquet M, Robaire B and Boerboom D: Seminiferous tubule degeneration and infertility in mice with sustained activation of WNT/CTNNB1 signaling in sertoli cells. Biol Reprod 79: 475-485, 2008.

38. Takase HM and Nusse R: Paracrine Wnt/ $\beta$-catenin signaling mediates proliferation of undifferentiated spermatogonia in the adult mouse testis. Proc Natl Acad Sci USA 113: E1489-E1497, 2016.

39. Kerr GE, Young JC, Horvay K, Abud HE and Loveland KL: Regulated Wnt/beta-catenin signaling sustains adult spermatogenesis in mice. Biol Reprod 90: 3, 2014.

40. Tanimoto K, Suzuki K, Jokitalo E, Sakai N, Sakaguchi T, Tamura D, Fujii G, Aoki K, Takada S, Ishida R, et al: Characterization of YIPF3 and YIPF4, cis-golgi localizing yip domain family proteins. Cell Struct Funct 36: 171-185, 2011.

41. Kranjc T, Dempsey E, Cagney G, Nakamura N, Shields DC and Simpson JC: Functional characterisation of the YIPF protein family in mammalian cells. Histochem Cell Biol 147: 439-451, 2017.

42. Kim SW, Jung YS, Ahn JB, Shin ES, Jang HW, Lee HJ, Kim T, Kim DY, Bang D, Kim WH and Cheon JH: Identification of genetic susceptibility loci for intestinal Behcet's disease. Sci Rep 7: 39850, 2017.

43. Kang HW, Yoon HY, Ha YS, Kim WT, Kim YJ, Yun SJ, Lee SC and Kim WJ: FAM70B as a novel prognostic marker for cancer progression and cancer-specific death in muscle-invasive bladder cancer. Korean J Urol 53: 598-606, 2012.

44. Walenta L, Fleck D, Fröhlich T, von Eysmondt H, Arnold GJ, Spehr J, Schwarzer JU, Köhn FM, Spehr M and Mayerhofer A: ATP-mediated events in peritubular cells contribute to sterile testicular inflammation. Sci Rep 8: 1431, 2018.

45. Jurga AM, Piotrowska A, Makuch W, Przewlocka B and Mika J: Blockade of P2X4 receptors inhibits neuropathic pain-related behavior by preventing MMP-9 activation and, consequently, pronociceptive interleukin release in a rat model. Front Pharmacol 8: 48, 2017.

46. Tsuda M, Masuda T, Tozaki-Saitoh H and Inoue K: P2X4 receptors and neuropathic pain. Front Cell Neurosci 7: 191, 2013

47. Lalisse S, Hua J, Lenoir M, Linck N, Rassendren F and Ulmann L: Sensory neuronal P2RX4 receptors controls BDNF signaling in inflammatory pain. Sci Rep 8: 964, 2018.

48. Zech A, Wiesler B, Ayata CK, Schlaich T, Dürk T, Hoßfeld M, Ehrat N, Cicko S and Idzko M: P2rx4 deficiency in mice alleviates allergen-induced airway inflammation. Oncotarget 7 : 80288-80297, 2016.

49. Sadovnick AD, Gu BJ, Traboulsee AL, Bernales CQ, Encarnacion M, Yee IM, Criscuoli MG, Huang X, Ou A, Milligan CJ, et al: Purinergic receptors P2RX4 and P2RX7 in familial multiple sclerosis. Hum Mutat 38: 736-744, 2017.

This work is licensed under a Creative Commons Attribution-NonCommercial-NoDerivatives 4.0 International (CC BY-NC-ND 4.0) License. 